\title{
Paranasal sinus osteoma: is there any association with anatomical variations?*
}

\author{
Aleksa Janovic ${ }^{1,2}$, Svetlana Antic ${ }^{1,2}$, Zoran Rakocevic ${ }^{1}$, Marija Djuric ${ }^{2}$ \\ 'Department of Radiology, School of Dentistry, University of Belgrade, Belgrade, Serbia \\ ${ }^{2}$ Laboratory for Anthropology, Department of Anatomy, School of Medicine, University of Belgrade, Belgrade, Serbia
}

Rhinology 51: 000-000, 2013 DOI:10.4193/Rhino12.130

*Received for publication: August 3, 2012

Accepted: October 11, 2012

\section{SUMMARY}

Background: Developmental disturbances of the paranasal sinuses are proposed as the cause of osteoma. We examined whether such disturbances may result in the frequent presence of anatomical variations of the paranasal sinuses in patients with osteoma.

Methodology/Principal: The study was performed retrospectively on 2,820 patients subjected to CT examination during 2005 - 2011. Demographic and CT characteristics of osteoma, and associated pathological findings were evaluated for 104 patients with diagnosed osteoma. The presence of anatomical variations was assessed for 51 osteoma patients with a complete medical history, and for 1,233 patients from a control group.

Results: The prevalence of osteomas was found to be 3.69\%, with male to female ratio 1.08:1. The frontal sinus was most commonly affected. The presence of anatomical variations was more frequent in patients with osteoma than in controls, with significant differences confirmed for the sphenomaxillary plate, infraorbital cell, and crista galli pneumatization.

Conclusions: The paranasal sinus osteoma is associated with higher prevalence of anatomical variations. This can be explained either by the stronger influence of genetic and/or environmental factors on the development of the paranasal sinuses in patients with osteoma, or by their higher susceptibility to abovementioned factors.

Key words: paranasal sinus osteoma, anatomical variations, computed tomography

\section{Introduction}

Paranasal sinus osteoma is a benign osteogenic lesion, often diagnosed as an incidental radiological finding. The prevalence among patients subjected to the standard radiography and computed tomography examination is reported to be around $1 \%$ and $3 \%$, respectively ${ }^{(1-4)}$. Male individuals are generally more affected than females, with the male to female ratio 1.5 - 2.6:1 ${ }^{(5-7)}$. The most commonly involved site is the frontal sinus with $60-96 \%$ of the diagnosed osteomas ${ }^{(1,8-10)}$. Although the majority is asymptomatic, their clinical importance lies in the possibility to cause various complications ${ }^{(6,11)}$.

The etiology of osteoma is still doubtful. In addition to the proven influence of genetic factors on the development of multiple osteomas in Gardner's syndrome ${ }^{(11)}$, the cause of solitary osteoma is unknown. The proposed explanations such as trauma, inflammation, embryological factors, calcification of a polypus, metaplasia, and alteration of the calcium metabolism ensued mainly from the clinical assessment of individual cases ${ }^{(12,13)}$. Despite the fact that none of the proposed hypothesis has been proven ${ }^{(13)}$, there is a tendency in the literature to explain the etiology of paranasal osteomas by traumatic, infectious or developmental (embryonic) theories.

According to the traumatic theory, deregulation of bone remodeling caused by trauma leads to osteoma development ${ }^{(14)}$. 
The suggestion was based on a small number of patients, who have suffered facial or even surgical trauma before the diagnosis of paranasal sinus osteoma ${ }^{(9,15-17)}$. The period of bone growth during puberty had been recommended as critical for the development of trauma-induced osteomas ${ }^{(9)}$. The theory explained male preponderance due to their greater exposure to trauma ${ }^{(18)}$. The infectious theory proposes that chronic inflammation plays a key role in the development of osteomas ${ }^{(19)}$. The persistent irritation acts as a trigger for the proliferation of osteoblasts in the mucoperiosteum and subsequent increase in the osteogenic activity, which results in osteoma formation ${ }^{(20-23)}$. However, some authors believe that sinusitis occurs due to osteoma induced improper sinus drainage ${ }^{(6,7)}$.

The majority of osteomas of unknown cause have been attributed to developmental anomalies. Based on this embryonic theory, osteomas arise from osseous proliferation at the junction where the cartilaginous ethmoid bones meet the membranous frontal bones ${ }^{(24,25)}$. In favour of this theory is that osteomas are frequently diagnosed at the fronto-ethmoidal junction ${ }^{(24,26)}$. However, this theory cannot explain osteomas in maxillary and sphenoid sinuses.

Similarly to osteoma, it has been reported that both genetic and environmental factors influence the development of anatomical variations of the paranasal sinuses ${ }^{(27,28)}$. Studies on monozygotic and dizygotic twins have suggested that the appearance of certain anatomical variations is genetically determined, while other anomalies develop under the influence of the environmental factors ${ }^{(27,28)}$. However, there are no suggestions whether the same developmental disturbances responsible for osteoma development would possibly result in the frequent presence of anatomical variations in these patients.

Despite the fact that epidemiological and radiological characteristics of the paranasal sinus osteomas have been reported in numerous studies, there are no such data published for the Serbian population in recent literature.

The aim of this study was to evaluate demographic and radiological characteristics of paranasal sinus osteomas in patients from Serbia. The presence of anatomical variations was also evaluated in this patient group to reveal a possible association with osteomas.

\section{Materials and methods}

Study design

The study was performed on 2,820 patients subjected to CT examination due to suspected sinus disease in the period 2005 - 2011. Radiological examination was performed using Computed Tomography (Siemens Somatom Sensation 16) at the Department of Radiology, Faculty of Dentistry, Belgrade, Serbia.
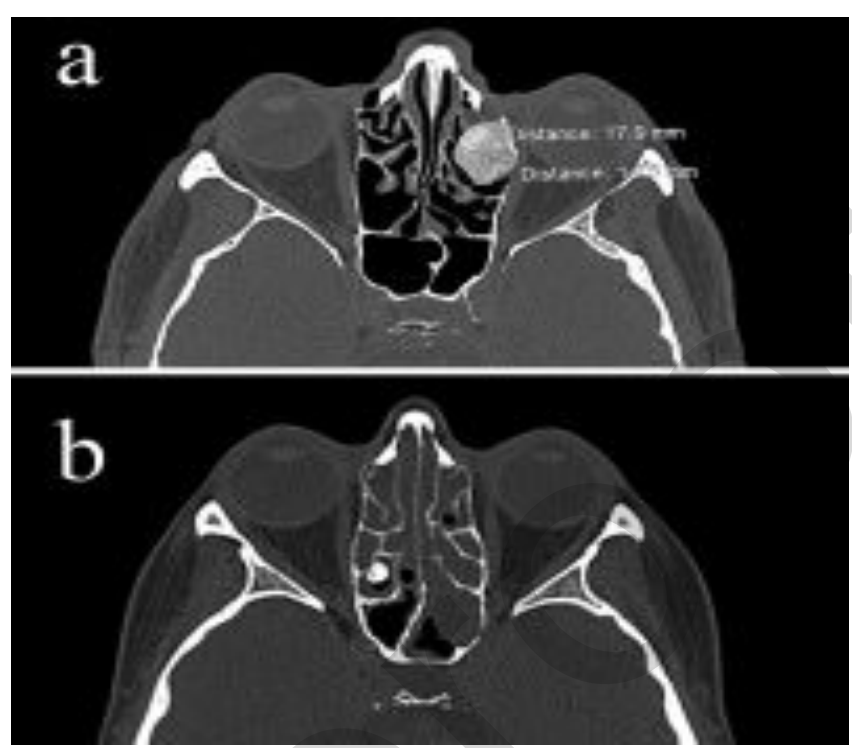

Figure 1. Axial computed tomography scans (bone window) show large osteoma in the left ethmoid sinus (a), and right-sided ethmoid osteoma associated with polyposis (b).

Paranasal sinuses in each patient were scanned in $3 \mathrm{~mm}$ thick axial sections and then reconstructed in the coronal plane with slice thickness of $0.75 \mathrm{~mm}$. All patients were examined by the same radiologist, the coauthor of this paper (Z.R.).

Paranasal sinus osteoma was diagnosed in 104 patients. Demographic characteristics (age and gender distribution), frequency of osteoma, CT characteristics of osteoma (location, side), and the presence of associated pathological findings were obtained from radiological reports that were available for all patients. Further analyses were performed on CT scans of 51 patients examined after June 2008, while the storage of CT documentation in our department started after this date. Scans of this patient group were reexamined to obtain data of osteoma size, shape, structure, and density. Size of osteoma was measured on axial slices (Figure 1a). Structure of osteoma was evaluated according to Earwaker ${ }^{(1)}$. The distance of osteoma from fronto-ethmoidal junction was measured on coronal sections for frontal and ethmoid sinus osteomas. The presence of the following anatomical variations was evaluated: supraorbital ethmoidal cell, sphenoethmoid cell, infraorbital ethmoidal cell, sphenomaxillary plate, pneumatization of the anterior clinoid process, pterygoid pneumatization, crista galli pneumatization, and concha bullosa. The variation was counted as present if it was observed on at least one side, except for the crista galli pneumatization. The level difference between cribriform plate and ethmoid roof was also measured.

A group of 1,233 patients without diagnosed osteoma, whose 
CT scans are also stored in our department during the abovementioned period, were introduced as a control group. Patients with a history of acute trauma, surgical intervention, neoplastic process, or extensive pathology that altered sinonasal anatomy were excluded from the study. The presence of the same anatomical variations was also evaluated for the control group of patients.

\section{Statistical analysis}

Data were analysed using SPSS 15 (Statistical Package for the Social Sciences). Parametric data were compared by means of Students't test or Mann-Whitney test, depending on the normality of the data distribution. Fisher's exact test was used for qualitative data analysis.

\section{Results}

Paranasal sinus osteoma was diagnosed in 104 (3.69\%) patients examined during 2005-2011. Patients were between 18 and 81 years old at the moment of diagnosis (mean age $49.5 \pm 14.3$ years). Males $(54 ; 51.9 \%)$ were slightly more affected than females $(50 ; 48.1 \%)$, with male to female ratio $1.08: 1$. Frontal sinus was the most common location of osteomas $(71 ; 68.3 \%)$, followed by the ethmoid sinuses $(29 ; 27.9 \%)$. In four patients, osteoma was diagnosed either in the maxillary sinus (1 case; $1.0 \%$ ) or in the sphenoid sinus (3 cases; $2.9 \%$ ). Right-sided paranasal sinus osteomas were more frequent (50.9\%) than left-sided (35.6\%). Nine patients had osteoma placed in the sagittal plane (8.7\%), whereas bilateral osteomas were detected in 5 cases (4.8\%). None of the patient had paranasal sinus osteoma(s) associated with Gardner syndrome. In one patient, osteoma was surgically removed due to intraorbital complications (Figure 1a). There were no other complications detected on computed tomography.

In the same sinus where osteoma was located, associated pa- thological conditions were present in 34 (32.7\%) patients (Table 1). The pathological conditions include catarrhal sinusitis (25; $24.0 \%)$, polyposis $(5 ; 4.9 \%)$, and generalized inflammation (4; $3.8 \%$ ) in descending order (Figure $1 \mathrm{~b}$ ).

The other parameters were evaluated in the group of 51 patients with an average age of $50.3 \pm 13.4$ years (range 18 - 81 years). Fifty-four osteomas were diagnosed in these individuals ( 3 were bilateral). Osteomas were dominantly oval in shape (33 cases; $61.1 \%)$, whereas a similar number of round $(11 ; 20.4 \%)$ and irregular $(10 ; 18.5 \%)$ osteomas was observed. The size of osteomas varied from $2.2 \mathrm{~mm}$ to $17.9 \mathrm{~mm}$ at their greatest points with the mean diameter $8.07 \pm 4.04 \mathrm{~mm}$. The majority of osteomas were composed of uniformly sclerotic bone (53.7\%). The remaining cases showed the heterogeneous structure without (17, 31.5\%) or with well-defined cortical shell (5 osteomas; 9.3\%). Only two osteomas (3.7\%) had cancellous structure, while target like appearance was observed in one case (1.8\%). Mean osteoma density was $1,171.1 \pm 387.4 \mathrm{HU}$, ranging from $471.1 \mathrm{HU}$ to 1791.0 HU. Out of 51 osteomas (including cases with bilateral osteomas) located in frontal and/or ethmoid sinuses, there were 18 (35.3\%) located in the region of fronto-ethmoidal junction. In other fronto-ethmoid osteomas the distance from the fronto-ethmoidal junction varied up to $70.8 \mathrm{~mm}$, with the mean distance $9.9 \pm$ $5.8 \mathrm{~mm}$.

The control group consisted of a similar number of males (48.7\%) and females (51.3\%), with a mean age of $49.2 \pm 16.1$ years. Statistical analysis showed no significant age $(t=-0.262$; $p>0.05$ ) and gender (Fisher's Exact Test, $p>0.05$ ) differences between two patient groups.

The presence of anatomical variations in osteoma group and controls is summarized in Table 2. Concha bullosa was the most

Table 1. Pathological findings in paranasal sinuses associated with osteoma.

\begin{tabular}{lccccc} 
Associated pathology & $\begin{array}{c}\text { Frontal } \\
(\mathbf{n}=\mathbf{7 1})\end{array}$ & $\begin{array}{c}\text { Ethmoid } \\
(\mathbf{n}=\mathbf{2 9})\end{array}$ & $\begin{array}{c}\text { Maxillary } \\
(\mathbf{n}=\mathbf{1})\end{array}$ & $\begin{array}{c}\text { Sphenoid } \\
(\mathbf{n}=\mathbf{3})\end{array}$ & $\begin{array}{c}\text { Total } \\
(\mathbf{n}=104)\end{array}$ \\
\hline None & $56(78.8 \%)$ & $11(41.4 \%)$ & $1(100 \%)$ & $2(66.7 \%)$ & $25(24.0 \%)$ \\
Catarrhal sinusitis & $14(19.7 \%)$ & $10(34.5 \%)$ & - & $1(33.3 \%)$ & $5(4.9 \%)$
\end{tabular}



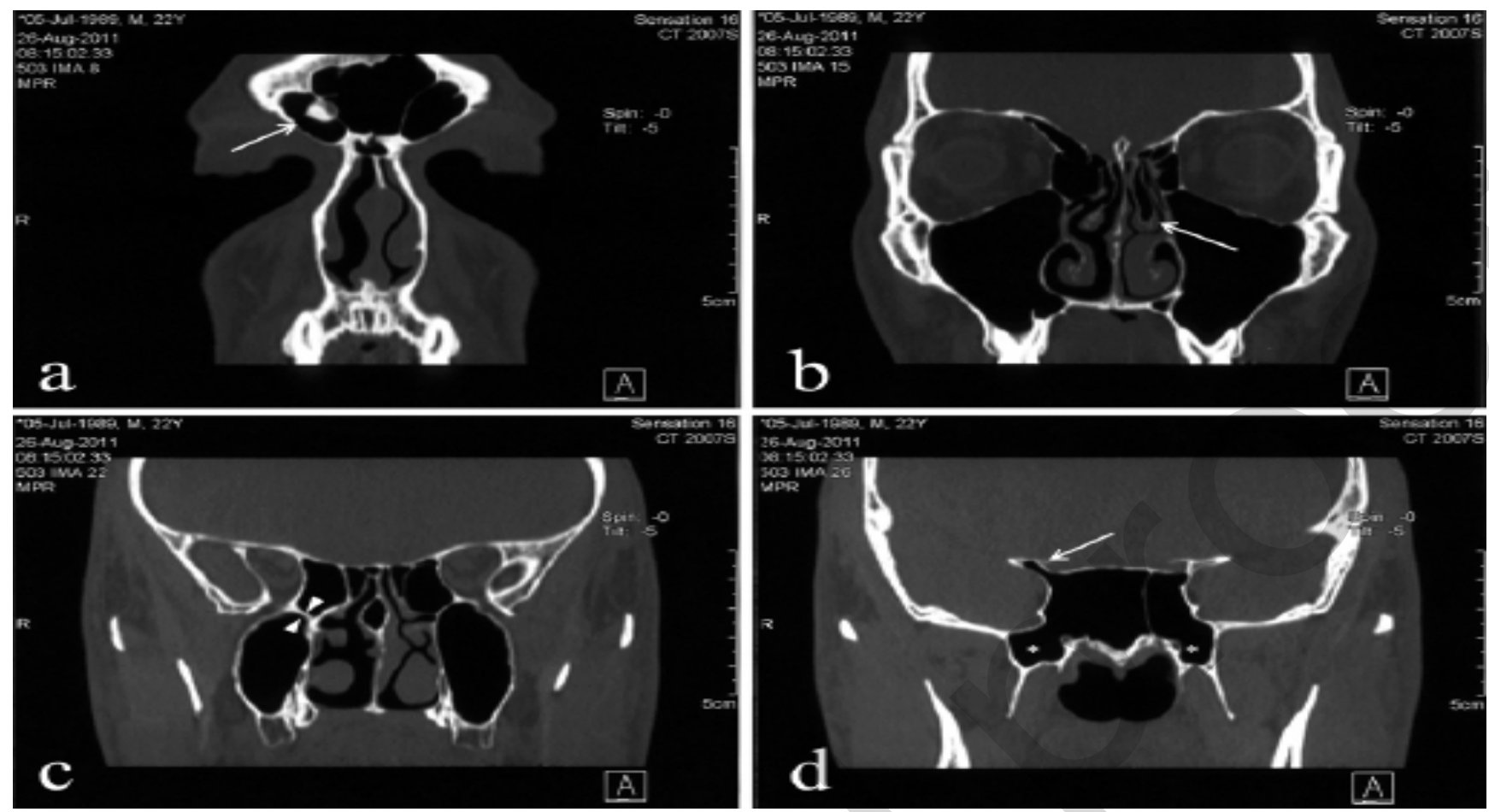

Figure 2. Coronal CT scans of a male patient show the frontal sinus osteoma (a) coexistent with the concha bullosa (b), sphenomaxillary plate (c), pneumatized pterygoid processes ( $d$, asterisk), and pneumatized the anterior clinoid process ( $d$, arrow).

Table 2. The presence of anatomical variations in patients with osteoma and control group.

\begin{tabular}{|c|c|c|c|}
\hline Anatomical variation & $\begin{array}{c}\text { Osteoma group } \\
(n=51)\end{array}$ & $\begin{array}{l}\text { Control group } \\
(n=1233)\end{array}$ & Statistical analysis \\
\hline Supraorbital ethmoidal cell & $2(3.9 \%)$ & $23(1.9 \%)$ & $p>0.05^{*}$ \\
\hline Sphenomaxillary plate & $12(23.5 \%)$ & $105(8.5 \%)$ & $\mathrm{p}<0.01^{*}$ \\
\hline Sphenoethmoid cell & $2(3.9 \%)$ & $61(4.9 \%)$ & $p>0.05^{*}$ \\
\hline $\begin{array}{l}\text { Pneumatization of anterior clinoid } \\
\text { process }\end{array}$ & $6(11.8 \%)$ & $67(5.4 \%)$ & $p>0.05^{*}$ \\
\hline Concha bullosa & $19(37.3 \%)$ & $348(28.2 \%)$ & $p>0.05^{*}$ \\
\hline Infraorbital ethmoidal cell & $8(15.7 \%)$ & $36(2.9 \%)$ & $p<0.01$ \\
\hline Pterygoid pneumatization & $18(35.3 \%)$ & $310(25.1 \%)$ & $p>0.05^{*}$ \\
\hline Crista galli pneumatization & $8(15.7 \%)$ & $63(5.1 \%)$ & $\mathrm{p}<0.01^{*}$ \\
\hline $\begin{array}{l}\text { Level difference between cribri- } \\
\text { form plate and ethmoid roof (in } \\
\mathrm{mm} \text { ) } 5.0 \pm 1.7\end{array}$ & $5.4 \pm 2.1$ & $5.0 \pm 1.7$ & $Z=-1.282 ; p>0.05^{* *}$ \\
\hline
\end{tabular}

\footnotetext{
* - Fisher's Exact Test, ${ }^{* *}$ - Mann-Whitney Test
} 
commonly observed variation in both groups, followed by pterygoid pneumatization and sphenomaxillary plate (Figure 2). The presence of the majority of anatomical variations was generally more frequent in patients with osteoma (Table 2). The same was observed for the level distance between cribriform plate and ethmoid roof. Only the sphenoethmoidal cell was more commonly seen in controls. The presence of sphenomaxillary plate, infraorbital cell, and crista galli pneumatization was found to vary significantly between groups (Table 2). Observed differences in the presence of other anatomical variations were not reached statistical significance.

\section{Discussion}

Data relating to the demographic characteristics of osteomas in Serbian population are scarce. Among the few published papers, Udovicki et al., described 30 cases of paranasal sinus osteomas treated during the period 1960-1989 (29), while Savic and Djeric proposed indications for surgical removal of sinus osteoma in 61 patients ${ }^{(30)}$. The authors reported a slight male predominance and the frontal sinus as the most common location of osteoma. Our results related to gender distribution and location of osteomas are consistent with data from literature ${ }^{(1,4,7,30)}$. However, we found a slightly higher prevalence of osteomas (3.69\%) in comparison with the average prevalence of $3 \%{ }^{(1,7)}$. This can be internreted as an incidental finding rather than a real increase in the prevalence of osteoma in the Serbian population. In general, the number of patients subjected to radiological examination due to chronic sinusitis increases worldwide and thus the possibility to detect osteoma ${ }^{(31)}$. The increasing prevalence of osteoma has been reported in the last few decades along with the development of imaging technique used for diagnosis ${ }^{(6)}$. In the era when plain radiography was the main diagnostic tool, the reported prevalence increased from $0.4 \%$ to $1 \%{ }^{(1,6)}$. However, after CT was established as a method of choice for the diagnosis of the paranasal sinus diseases ${ }^{(31)}$, the prevalence increased to $3 \%{ }^{(6,7)}$. Moreover, osteomas are most commonly encountered in fourth decade ${ }^{(4)}$, which is also the peak incidence of chronic sinusitis ${ }^{(31)}$.

Large population studies showed sinusitis coexistent with osteoma in around one third of the patients ${ }^{(7)}$. In our sample, osteomas were accompanied by pathological findings in sinuses in a similar percentage (Table 1). However, the exact causal relationship between osteoma and sinus inflammation has not been established yet. According to the first hypothesis, the size of osteoma and/or its location near sinus ostia influence the improper sinus drainage and the consequent development of inflammation ${ }^{(6)}$. Radiological evaluation by means of CT may be helpful to support this hypothesis. There are also opposite suggestions in the literature. Namely, chronic sinus inflam- mation may act as a trigger for bone proliferation and consequent osteoma development ${ }^{(19-23)}$. It is more difficult to discern clinically whether the osteoma occurred primary or secondary in the cases with coexistent chronic inflammation, particularly in patients with small osteomas subjected to the CT examination for the first time. Erdogan et al. reported osteoma accompanied by polyposis in around $28 \%{ }^{(7)}$. In the present study, CT revealed polyposis in only five (4.9\%) patients. Given that osteoblasts need time to form an osteoma of a few $\mathrm{mm}$ in size, it seems more likely that osteoma was present before development of chronic inflammation. This is supported by the fact that majority of osteomas are clinically silent and represent coincidental finding on radiographies ${ }^{(1)}$.

Facial trauma during bone growth in adolescence has been suggested to interfere with bone remodeling, which leads to osteoma development ${ }^{(14)}$. Greater exposure to trauma in males was also implicated in the traumatic theory ${ }^{(18)}$. Our results, however, showed almost equal affection of both sexes. Given that the data related to the history of facial trauma during adolescence were missing, the role of trauma in the present study can not be estimated with accuracy.

The embryological theory assumes osteomas as a consequence of disturbances during bone development. The region of the fronto-ethmoidal junction was considered as the main location where such disturbances occur due to close relationship between the bones with different ossification patterns. The theory was used to explain the appearance of fronto-ethmoid osteomas. However, out of total 51 fronto-ethmoid osteomas diagnosed in our patients, only 18 (35.3\%) were located at the level of the fronto-ethmoidal junction. Many authors also showed that a significant number of fronto-ethmoid osteomas do not arise from the junction ${ }^{24,32)}$. Nevertheless, the etiology of osteoma is most commonly attributed to the embiological factors.

Both genetic and environmental factors have been also reported to influence the development of anatomical variations of the paranasal sinuses. Chaiyasate et al., suggested a genetic influence in the development of concha bullosa due to its more frequent presence in monozygotic twins than in dizygotic twins. In the general population, concha bullosa is the commonest anatomic variation after nasal septal deviation ${ }^{(3,34)}$. In our sample, this variation was also the most frequent in both groups, with the higher percent detected in the osteoma group (Figure 2b). However, observed difference was not statistically significant (Table 2). Pneumatization of the pterygoid processes (Figure $2 \mathrm{~d}$ ) and the sphenomaxillary plate (Figure 2c) were also dominant in both groups, with significant difference detected in the last (Table 2). The difference could possibly be attributed 
to variations in the degree of sinus pneumatization. The state is supported by the fact that the osteoma group had also significantly higher percent of the pneumatized crista galli. Although there are no suggestions which of the proposed factors are dominant in the development of these anatomical variations, the degree of the sinus pneumatization has been addressed to genetic factors ${ }^{(27)}$. Probable genetic influence was also reported for the level distance between the cribriform plate and the ethmoid roof, and for the bilateral presence of the supraorbital cell type I and II (27). Although the higher frequency of the supraorbital cell and the greater values of the level distance were recorded in patients with osteoma, the differences were not reached statistical significance when compared with the control group (Table 2).

Environmental factors were suggested to play a dominant role in the development of the septal deviation, infraorbital cell, and the supraorbital cell type III and IV ${ }^{(27,28)}$. Facial trauma has been recognized as one of the environmental factors that causes nasal septal deviation ${ }^{(28)}$. However, the presence of this variation was not evaluated in our study, since the data related to the history of the facial trauma were not available. The groups differed significantly in the presence of the infraorbital cell, which was more frequent in the osteoma group (Table 2). This finding may indicate that patients with osteoma were under greater exposure of the same environmental factors that also influence the development of the infraorbital cell in the control group. The same could be hypothesized for the genetically determined development of the sphenomaxillary plate and the crista galli pneumatization. It is also possible that patients with osteoma were more susceptible to the same genetic and environmental factors that caused the development of the anatomical variations in both groups.

Although our results are limited to a small number of patients, clinicians should be aware of the possible association between osteoma and anatomical variations. Their coexistence is of a special clinical importance if the patient has to be treated surgically due to osteoma related complications ${ }^{(6)}$. Further studies on larger series are needed to clarify the exact nature of the association between osteoma and the anatomical variations.

In conclusion, our results suggest that patients with osteoma develop anatomical variations of the paranasal sinuses more frequently than patients without osteoma. The paranasal sinus osteoma was associated with the sphenomaxillary plate, infraorbital cell, and crista galli pneumatization, which all showed significantly higher prevalence in osteoma group in comparison with controls. The association could be explained either by a stronger influence of the genetic and environmental factors on the development of the paranasal sinuses in patients with osteoma, or by their higher susceptibility to both abovementioned factors. The presence of the anatomical variations is of special clinical importance if the osteoma has to be surgically removed.

\section{Acknowledgements}

The authors acknowledge support from the Ministry of Science of the Republic of Serbia, project number 45005.

\section{Authorship contribution}

$A J$ and SA made the research question, reviewed the literature, and wrote the majority of the manuscript. ZR examined all the patients, reviewed their scans and radiological reports, and wrote one part of the materials and method, and the results section. MD is head of the project, contributed to the study design and correct interpretation of the results.

\section{Conflict of interest}

None

\section{References}

1. Earwaker J. Paranasal sinus osteomas: a review of 46 cases. Skeletal Radiol. 1993 22: 417-423.

2. Vowles RH, Bleach NR. Frontoethmoida osteoma. Ann Otol Rhinol Laryngol. 1999; 108: 522-524.

3. Eller $R$, Sillers M. Common fibro-osseous lesions of the paranasal sinuses. Otolaryngol Clin North Am. 2006; 39: 585600.

4. Lund V, Stammberger $H$, Nicolai $P$, Castelnuovo P. European position paper on endoscopic management of the nose, paranasal sinuses and skull base. Rhinology Supplement. 2010; 22: 1-144.

5. Atallah $\mathrm{N}$, Jay M. Osteomas of the paranasal sinus. J Laryngol Otol. 1981; 95: 291-304.

6. Mansour AM, Salti H, Uwaydat S, Dakroub $R$, Bashshour Z. Ethmoid sinus osteoma presenting as epiphora and orbital cellulitis: case report and literature review. Surv Ophthalmol. 1999; 43: 413-426.

7. Erdogan N, Demir U, Songu M, et al. A prospective study of paranasal sinus osteomas in 1,889 cases: changing patterns of localization. Laryngoscope. 2009; 119: 2355-2359.

8. Nielsen GP, Rosenberg AE. Update on bone forming tumors of the head and neck. Head Neck Pathol. 2007; 1: 87-93.

9. Morreti A, Croce A, Leona O, D'agostino L. Osteoma of the maxillary sinus: case report. Acta Otorhinolaryngolyngol Ital. 2004; 24 219-222.

10. Zouloumis L, Lazaridis N, Papadaki M, Epivatianos A. Osteoma of the ethmoid sinus: a rare case of recurrence. $\mathrm{Br} J$ of Oral Maxillofac Surg. 2005; 43: 520-522.

11. Folpe $A L$, Inwards $C Y$. Bone and soft tissue pathology. Saunders, 2009.
12. Namdar I, Edelstein DR, Huo J, et al. Management of osteomas of the paranasal sinuses. Am J Rhinol. 1998; 12: 393-398.

13. Furlanetto EC, Rocha JR, Heitz C. Osteoma of the zygomatic arch - report of a case. Int J Oral Maxillofac Surg. 2004; 33: 310-311.

14. Sudhoff $H$, Theegarten D, Luckhaupt $H$. Osteoma of the maxillary sinus. Laryngorhinootologie. 2001; 80: 275-277.

15. Childrey $\mathrm{JH}$. Osteoma of sinuses, the frontal and sphenoid bone: report of fifteen cases. Arch Otolaryngol. 1939; 30: 63-72.

16. Rawlings AG. Osteoma of the maxillary sinus: report of a case. Arch Otolaryngol. 1940; 32: 499-505.

17. Kim YJ, Kim DY, Jun YJ, Seo BC. Ethmoid sinus osteoma associated with blow-out fracture. J Craniofac Surg. 2012; 23: 477-479.

18. Appalanarasayya K, Murthy AS, Viswanath CK, Devi OB. Osteoma involving the orbit: 
<smiles>C1#CCC=C1</smiles> 